\title{
Erratum to: National trends in incidence and outcomes of abdominal aortic aneurysm among elderly type 2 diabetic and non-diabetic patients in Spain (2003-2012)
}

Ana Lopez-de-Andrés ${ }^{1 *}$, Isabel Jiménez-Trujillo', Rodrigo Jiménez-García', Valentín Hernández-Barrera', José Ma de Miguel-Yanes ${ }^{2}$, Manuel Méndez-Bailón ${ }^{3}$, Napoleón Perez-Farinos ${ }^{4}$, Miguel Ángel Salinero-Fort ${ }^{5}$ and Pilar Carrasco-Garrido ${ }^{1}$

\section{Erratum to: Cardiovascular Diabetology (2015) 14:48 \\ DOI 10.1186/s12933-015-0216-1}

After publication of the original article [1], it came to the authors' attention that a source of funding for the study presented had been inadvertently omitted. The Acknowledgements section should therefore have read as follows:

"This study was funded by the FIS (Fondo de Investigaciones Sanitarias-Health Research Fund, Grant No. PI13/00118, Instituto de Salud Carlos III), co-financed by the European Union through the Fondo Europeo de Desarrollo Regional (FEDER, "Una manera de hacer Europa"), and by the Grupo de Excelencia Investigadora URJC-Banco Santander No. 30VCPIGI03: Investigación traslacional en el proceso de salud-enfermedad (ITPSE)."
The online version of the original article can be found under doi:10.1186/s12933-015-0216-1.

Published online: 21 July 2016

Reference

1. Lopez-de-Andrés A, Jiménez-Trujillo I, Jiménez-García R, HernándezBarrera V, de Miguel-Yanes JM, Méndez-Bailón M, Perez-Farinos N, Salinero-Fort MÁ, Carrasco-Garrido P. National trends in incidence and outcomes of abdominal aortic aneurysm among elderly type 2 diabetic and non-diabetic patients in Spain (2003-2012). Cardiovasc Diabetol. 2015;14:48. doi:10.1186/s12933-015-0216-1.

\begin{abstract}
Author details
${ }^{1}$ Preventive Medicine and Public Health Teaching and Research Unit, Health Sciences Faculty, Rey Juan Carlos University, Avda. de Atenas s/n, 28922, Alcorcon, Comunidad de Madrid, Spain. ${ }^{2}$ Medicine Department, Hospital Gregorio Marañon, Madrid, Comunidad de Madrid, Spain. ${ }^{3}$ Medicine Department, Hospital Clínico San Carlos, Madrid, Comunidad de Madrid, Spain. ${ }^{4}$ Health Security Agency, Ministry of Health, Madrid, Comunidad de Madrid, Spain. ${ }^{5}$ Dirección Técnica de Docencia e Investigación, Gerencia Atención Primaria, Madrid, Comunidad de Madrid, Spain.
\end{abstract}

*Correspondence: ana.lopez@urjc.es

1 Preventive Medicine and Public Health Teaching and Research Unit, Health Sciences Faculty, Rey Juan Carlos University, Avda. de Atenas s/n, 28922, Alcorcon, Comunidad de Madrid, Spain 